Pacific Journal of Mathematic 


\title{
INVERSE LIMITS AND MAPPINGS OF MINIMAL TOPOLOGICAL SPACES
}

\author{
Louis M. Friedler ANd Dix H. Pettey
}

\begin{abstract}
S. W. Willard has conjectured that every $H$-closed space is the continuous image of a minimal Hausdorff space. In this paper we verify Willard's conjecture and show as well that every $R$-closed space is the continuous image of a minimal regular space. We also identify conditions sufficient to guarantee that an $H$-closed space be the finite-to-one continuous image of a minimal Hausdorff space. We give an example of a nonvacuously $R(i i)$ space whose product with itself is neither $R(i)$ nor $R(i i)$, and we obtain a number of results concerning inverse limits of $H$-closed spaces and $R$-closed spaces.
\end{abstract}

1. Introduction. Throughout this paper, the word map (or mapping) will always mean a continuous function.

If $P$ is a topological property, then a $P$-space is called $P$-closed if it is closed in every $P$-space in which it is embedded and minimal $P$ if there is no strictly coarser $P$ topology on the same underlying set. For $P=$ Hausdorff $\left[P=\right.$ regular $T_{1}$ ] the $P$-closed and minimal $P$ properties will be denoted as $H$-closed [ $R$-closed] and $M H[M R]$. In studying mapping properties of $M H$ spaces, S. W. Willard [14] showed that the Hausdorff spaces whose Hausdorff continuous images are always $M H$ are precisely the functionally compact spaces of Dickman and Zame [4] and conjectured that the Hausdorff spaces which are the continuous images of $M H$ spaces are precisely the $H$ closed spaces. An analogous conjecture can be posed for $M R$ and $R$-closed spaces. Here we shall prove that every $H$-closed [ $R$-closed] space is the image, under an open and perfect mapping, of an $M H$ [MR] space of the same weight. Since every Hausdorff [regular $T_{1}$ ] continuous image of an $H$-closed [ $R$-closed] space is $H$-closed [ $R$-closed] (see [2]), we thereby establish both of the above mentioned conjectures. We also obtain results concerning products of $M R$ spaces and products of $R(i i)$ spaces (see $\S 2$ for definition) as well as a number of theorems concerning inverse limits of $H$-closed spaces and of $R$-closed spaces. In $\S 4$ we determine conditions that guarantee that an $H$-closed space be the image of an $M H$ space under an atmost-two-to-one mapping.

A set $V$ in a topological space is regularly open if $V=\operatorname{int} \bar{V}$. A space is semiregular at a point if that point has a neighborhood base of regularly open sets. A point of a topological space at which the space is semiregular will be called a semiregular point of the 
space. A space is said to be semiregular if it is semiregular at each of its points. It is well-known $[2,3.9]$ that a Hausdorff space is $M H$ if and only if it is $H$-closed and semiregular. The reader is referred to the survey paper [2] by Berri, Porter and Stephenson for the standard filterbase and covering characterizations of $H$-closed, $M H, R$-closed and $M R$ spaces and to [5] for information concerning inverse limits. A point of an $H$-closed [ $R$-closed] space will be called a bad point if it is the only cluster point of some nonconvergent open [regular] filterbase on the space. For an $H$-closed space, the bad points are precisely those points at which the space is not semiregular.

For an inverse system $\left\{Y_{\alpha} ; f_{\alpha}^{\beta}\right\}$, the following terminology and notation will be used: the mappings $f_{\alpha}^{\beta}: Y_{\beta} \rightarrow Y_{\alpha}$ will be called bonding maps, the inverse limit space will be denoted as $Y_{\infty}$, and the natural projection from $Y_{\infty}$ to the space $Y_{\alpha}$ will be denoted as $f_{\alpha}$. It will always be assumed that the system is indexed over a nonempty directed set and that for each $\alpha$ in the index set $f_{\alpha}^{\alpha}$ is the identity function on $Y_{\alpha}$.

We shall let $N$ denote the set of natural numbers.

If $\alpha$ and $\beta$ are ordinals then $[\alpha, \beta)$ will denote the set of all ordinals greater than or equal to $\alpha$ and less than $\beta$, while $[\alpha, \beta]$ will denote the set $[\alpha, \beta) \cup\{\beta\}$.

2. Open perfect mappings. In this section, no separation axioms will be assumed except where explicitly indicated. Furthermore, "regular" will not imply $T_{1}$.

The terms $H(i), R(i), H(i i)$ and $R(i i)$ will be used as in [10]; i.e., a topological space is $H(i)[R(i)]$ if every open filterbase [regular filterbase] has a cluster point and $H(i i)[R(i i)]$ if every open filterbase [regular filterbase] having a unique cluster point is convergent. For a Hausdorff space, the properties $H(i)$ and $H(i i)$ are equivalent, respectively, to the $H$-closed and $M H$ properties (see [2, 3.2 and 3.9]). Likewise, for a regular $T_{1}$ space, properties $R(i)$ and $R(i i)$ are equivalent, respectively, to the properties $R$-closed and $M R[2,4.14$ and 4.15]. A topological space is nonvacuously $H(i i)$ [nonvacuously $R(i i)]$ if it is $H(i i)[(R(i i)]$ and has at least one open filterbase [regular filterbase] with a unique cluster point. As is observed in [10], a space may be $H(i i)[R(i i)]$ without being $H(i)[R(i)]$. A nonvacuously $H(i i)$ [nonvacuously $R(i i)$ ] space is, however, necessarily $H(i)[R(i)]$. Furthermore, every continuous image of an $H(i)[R(i)]$ space is $H(i)$ [ $R(i)]$.

A topological space $X$ will be called strongly $H(i i)$ [strongly $R(i i)$ ] if there is a base $\mathscr{V}$ for $X$ such that every member of $\mathscr{V}$ has an $H(i)[R(i)]$ complement in $X$. It is easily verified that every strongly 
$H(i i)$ [strongly $R(i i)$ ] space is both $H(i)$ and $H(i i)$ [R(i) and $R(i i)$ ]. A topological space is said to be strongly minimal regular (abbreviated $S M R)$ if it is a strongly $R(i i)$, regular $T_{1}$ space.

REmark. R. M. Stephenson, Jr., introduced $S M R$ spaces to the literature in [13], and it is known that not all $M R$ spaces are $S M R$ (see [9]). This is in contrast to the situation for $M H$ spaces, for it is not difficult to show that every $M H$ space is strongly $H(i i)$.

For a topological space $X$ we shall let $w(X)$ denote the weight of $X$ (i.e., the smallest cardinal $\kappa$ such that $X$ has a base of cardinality $\kappa$ ).

A number of preliminary lemmas will be used in the proof of the main theorem (Theorem 2.8) of this section. Some of these are well-known results or immediate corollaries to well-known results and will therefore be given without detailed proofs.

LeMma 2.1. Let $\tau$ be an infinite initial ordinal. Then there is a one-to-one function $\sigma$ from $[0, \tau) \times[0, \tau)$ onto $[1, \tau)$ such that for each $(\alpha, \gamma)$ in $[0, \tau) \times[0, \tau), \sigma(\alpha, \gamma)>\alpha$.

Proof. Since $[1, \tau)$ and $[0, \tau) \times[0, \tau)$ are of the same cardinality, there is a one-to-one function $\phi$ from $[1, \tau)$ onto $[0, \tau) \times[0, \tau)$. For each $\alpha$ in $[1, \tau)$, let $\phi_{1}(\alpha)$ denote the first coordinate of $\phi(\alpha)$. We now define, inductively, a function $g$ from $[1, \tau)$ into $[1, \tau)$ as follows. Suppose that $\beta \in[1, \tau)$ and that for each $\alpha$ in $[1, \beta), g(\alpha)$ has been defined. Since $\tau$ is an initial ordinal, the set

$$
\left[\phi_{1}(\beta)+1, \tau\right)-\{g(\alpha) \mid \alpha \in[1, \beta)\}
$$

is nonempty. Define $g(\beta)$ to be the least member of this set. The function $g$ thus defined is clearly one-to-one. We assert that $g$ takes $[1, \tau)$ onto itself. For if $\lambda \in[1, \tau)$ then, since $\phi$ takes $[1, \tau)$ onto $[0, \tau) \times[0, \tau)$ and since the cardinality of $[1, \lambda)$ is less than that of $\{(\alpha, \gamma) \in[0, \tau) \times[0, \tau) \mid \alpha<\lambda\}$, the set $\left\{\beta \in[1, \tau) \mid g(\beta) \geqq \lambda, \phi_{1}(\beta)<\lambda\right\}$ must be nonempty. The least member of this set will necessarily be taken onto $\lambda$ by $g$. Now by defining $\sigma$ to be the function $g \phi^{-1}$, we obtain the required function from $[0, \tau) \times[0, \tau)$ onto $[1, \tau)$.

LEMMA 2.2. Let $\kappa$ be a cardinal and $\gamma$ an ordinal of cardinality less than or equal to $\kappa$. If an inverse system of topological spaces,

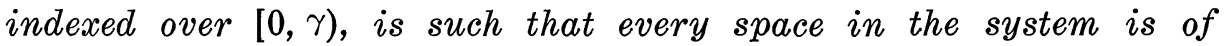
weight less than or equal to $\kappa$, then the inverse limit space is of weight less than or equal to $\kappa$. (This result follows easily from [5, 2.3(2), p. 428].) 
LEMMA 2.3. The inverse limit of Hausdorff [regular] spaces is a Hausdorff [regular] space. (This is an immediate consequence of the fact that the inverse limit is a subspace of the product of the spaces in the system.)

LEMMA 2.4. Let $\left\{Y_{\alpha} ; f_{\alpha}^{\beta}\right\}$ be an inverse system of topological spaces. If each bonding map is open and each projection $f_{\alpha}: Y_{\infty} \rightarrow Y_{\alpha}$ is onto, then each projection is open. (This follows easily from [5, 2.3(2), p. 428].)

Lemma 2.5. Let $\left\{Y_{\alpha} ; f_{\alpha}^{\beta}\right\}$ be an inverse system of topological spaces. If each bonding map is perfect and onto and has Hausdorff point inverses, then each projection $f_{\alpha}: Y_{\infty} \rightarrow Y_{\alpha}$ is perfect and onto and has Hausdorff point inverses.

Proof. Let $\mathscr{D}$ denote the directed set over which the system is indexed. For each $\alpha$ in $\mathscr{D}$ and each point $s$ of $Y_{\alpha}$, each of the sets $\left(f_{\alpha}^{\beta}\right)^{-1}(s)(\beta \geqq \alpha)$ is a nonempty compact Hausdorff space; therefore $\left(f_{\alpha}\right)^{-1}(s)$, which is homeomorphic to $\lim \left(f_{\alpha}^{\beta}\right)^{-1}(s)(\beta \geqq \alpha)$, is nonempty, compact and Hausdorff [5, 2.4, p. 429]. It remains only to be shown that each $f_{\alpha}$ is closed. Assume that for some $\beta$ in $\mathscr{D}$ and some closed set $K$ in $Y_{\infty}, f_{\beta}(K)$ is not closed in $Y_{\beta}$. Let $z$ be a point of $\mathrm{Cl} f_{\beta}(K)-f_{\beta}(K)$. Since $\left(f_{\beta}\right)^{-1}(z)$ is compact and does not intersect $K,[5,2.3(2)$, p. 428] implies that for some finite subset $\{\alpha(1), \alpha(2)$, $\cdots, \alpha(n)\}$ of $\mathscr{D}$ there are open sets $V_{1}, V_{2}, \cdots, V_{n}$ in $Y_{\alpha(1)}, Y_{\alpha(2)}, \cdots, Y_{\alpha(n)}$, respectively, such that $\left(f_{\beta}\right)^{-1}(z)$ is contained in $\mathrm{U}_{i=1}^{n}\left(f_{\alpha(i)}\right)^{-1}\left(V_{i}\right)$ and $\bigcup_{i=1}^{n}\left(f_{\alpha(i)}\right)^{-1}\left(V_{i}\right)$ does not intersect $K$. Choose $\gamma$ in $\mathscr{D}$ such that $\gamma \geqq \max \{\beta, \alpha(1), \alpha(2), \cdots, \alpha(n)\}$. Let $W_{\gamma}$ denote the set $\bigcup_{i=1}^{n}\left(f_{\alpha(i)}^{r}\right)^{-1}\left(V_{i}\right)$. Then $W_{r}$ is an open set in $Y_{r}$ such that $W_{r} \cap f_{r}(K)=\varnothing$ and such that $\left(f_{\beta}^{r}\right)^{-1}(z) \subset W_{r}$. But since $f_{\beta}^{r}$ is closed and $z$ is in the closure of $f_{\beta}^{\gamma} f_{\gamma}(K)$, this is impossible. Hence, we have a contradiction and our proof is complete.

LEMMA 2.6. Suppose that $\gamma$ is an ordinal greater than 0 and that $\left\{Y_{\alpha} ; f_{\alpha}^{\beta}\right\}$ is an inverse system over $[0, \gamma)$. Suppose, furthermore, that for each $\alpha$ in [0, $\gamma), K_{\alpha}$ is a subspace of $Y_{\alpha}$, and that for each $\beta$ in $[0, \gamma)$ and each $\alpha$ in $[0, \beta], f_{\alpha}^{\beta} \mid K_{\beta}$ is a homeomorphism from $K_{\beta}$ onto $K_{\alpha}$. If $K$ denotes the subspace $\cap\left(f_{\alpha}\right)^{-1}\left(K_{\alpha}\right)(a<\gamma)$ of $Y_{\infty}$, then for each $\alpha$ in $[0, \gamma), f_{n} \mid K$ is a homeomorphism from $K$ onto $K_{\alpha}$.

Proof. Since $K$ may be regarded as the inverse limit of the system $\left\{K_{\alpha} ; f_{\alpha}^{\beta} \mid K_{\beta}\right\}$, the desired conclusion follows as a result of basic properties of inverse systems. (See [5, p. 427].)

LEMMA 2.7. If $X$ is a topological space and if there exists an 
open perfect mapping from $X$ onto an $H(i)[R(i)]$ space, then $X$ is an $H(i)[R(i)]$ space. (The proof is routine and thus omitted.)

THEOREM 2.8. Let $Y$ be a topological space of infinite weight and $\tau$ the least ordinal of cardinality $w(Y)$. Then there exists an inverse system $\left\{Y_{\alpha} ; f_{\alpha}^{\beta}\right\}$ of topological spaces, indexed over $[0, \tau)$, such that

(1) $Y_{0}=Y$;

(2) if $Y_{0}$ is Hausdorff [regular] the then inverse limit space $Y_{\infty}$ is Hausdorff [regular];

(3) for each $\alpha$ in $[0, \tau)$; the projection $f_{\alpha}$ is open, perfect and onto;

(4) for each point $x$ of $Y_{\infty}$, there is a subspace $K$ of $Y_{\infty}$ such that $x \in K$ and $K$ is taken homeomorphically onto $Y$ by $f_{0}$;

(5) $w\left(Y_{\infty}\right)=w(Y)$;

(6) for each $\alpha$ in $[0, \tau)$ and each $H(i)[R(i)]$ subspace $S$ of $Y_{\alpha}$, $\left(f_{\alpha}\right)^{-1}(S)$ is a strongly $H(i i)$ [strongly $R(i i)$ ] subspace of $Y_{\infty}$.

Proof. By Lemma 2.1, there exists a one-to-one function $\sigma$ from $[0, \tau) \times[0, \tau)$ onto $[1, \tau)$ such that for each ordered pair $(\alpha, \gamma)$ in $[0, \tau) \times[0, \tau), \quad \sigma(\alpha, \gamma)>\alpha$. Using this function, we shall define inductively the inverse system $\left\{Y_{\alpha} ; f_{\alpha}^{\beta}\right\}$. For each $\beta$ in $[0, \tau)$ the space $Y_{\beta}$ will be chosen so that $w\left(Y_{\beta}\right) \leqq w(Y)$, and at the $\beta$ th stage of the induction we shall choose, in addition to the space $Y_{\beta}$ and the mappings $f_{a}^{\beta}(\alpha<\beta)$, a base $\left\{U_{\beta \gamma} \mid \gamma \in[0, \tau)\right\}$ for $Y_{\beta}$. We proceed as follows.

Let $Y_{0}=Y$ and let $f_{0}^{0}$ denote the identity mapping on $Y_{0}$. Choose a base $\left\{U_{0 r} \mid \gamma \in[0, \tau)\right\}$ for $Y_{0}$.

For each $\beta$ in $[1, \tau)$, let $Z_{\beta}$ denote the inverse limit of the system $\left\{Y_{\alpha} ; f_{\alpha}^{\gamma}\right\}_{0 \leqq \alpha \leqq \gamma<\beta}$, and for each $\alpha$ in $[0, \beta)$ let $\phi_{\alpha}^{\beta}$ denote the projection from $Z_{\beta}$ into $Y_{\alpha}$. Now let $(\lambda, \eta)=\sigma^{-1}(\beta)$. Then $\beta>\lambda$, so $U_{\lambda \eta}$ has already been chosen. Let $V_{\beta}=\left(\phi_{\lambda}^{\beta}\right)^{-1}\left(U_{\lambda \eta}\right)$ and define $Y_{\beta}$ to be the space obtained from $Z_{\beta} \times\{1,2\}$ by identifying $(z, 1)$ with $(z, 2)$ for each $z$ in $Z_{\beta}-V_{\beta}$. Let $f_{\beta}^{\beta}$ be the identity mapping on $Z_{\beta}$ and $p_{\beta}$ the natural projection from $Y_{\beta}$ onto $Z_{\beta}$. For each $\alpha$ in $[0, \beta)$, define $f_{\alpha}^{\beta}$ to be the function $\phi_{\alpha}^{\beta} p_{\beta}$. Then each $f_{\alpha}^{\beta}$ is continuous and, for $0 \leqq \alpha \leqq \gamma \leqq \beta$, we have $f_{\alpha}^{\beta}=f_{\alpha}^{\gamma} f_{\gamma}^{\beta}$. Finally, it follows from Lemma 2.2 that $w\left(Z_{\beta}\right) \leqq w(Y)$ and, consequently, that $w\left(Y_{\beta}\right) \leqq w(Y)$. Choose a base $\left\{U_{\beta \gamma} \mid \gamma \in[0, \tau)\right\}$ for $Y_{\beta}$.

By our construction, condition (1) of the conclusion of the theorem is immediately satisfied. To establish condition (2) it is sufficient, in light of Lemma 2.3, to show that if $Y_{0}$ is Hausdorff [regular] then $Y_{\alpha}$ is Hausdorff [regular] for each $\alpha$ in [0, $\tau$ ). This follows by induction, for if $0<\beta<\tau$ and $Y_{\alpha}$ is Hausdorff [regular] for each $\alpha$ 
in $[0, \beta)$ then by Lemma 2.3, $Z_{\beta}$ is Hausdorff [regular]. Since the Hausdorff [regular] property is certainly preserved by $\left(p_{\beta}\right)^{-1}, Y_{\beta}$ is also Hausdorff [regular].

We prove condition (3) in a similar manner. It follows by induction and the use of Lemmas 2.4 and 2.5 that for each $\beta$ in $[0, \tau)$ and each $\alpha$ in $[0, \beta], f_{\alpha}^{\beta}$ is open, perfect and onto and has Hausdorff point inverses. So, again by Lemmas 2.4 and 2.5 , each projection $f_{\alpha}$ is open, perfect and onto.

Condition (4) is established as follows. For each function $g$ from $[1, \tau)$ into $\{1,2\}$, let $K^{g}$ denote the set of all points $x$ of $Y_{\infty}$ such that for each $\alpha$ in $[1, \tau), f_{\alpha}(x)$ is in $Z_{\alpha} \times\{g(\alpha)\}$. Then for each $x$ in $Y_{\infty}$ there is some $\dot{g}$ such that $x \in K^{g}$. We shall now show that each $K^{g}$ is taken homeomorphically onto $Y\left(=Y_{0}\right)$ by $f_{0}$. For each $g(g:[1, \tau) \rightarrow$ $\{1,2\})$, let $K_{0}^{g}=Y_{0}$ and, for each $\beta$ in $[1, \tau)$, let $K_{\beta}^{g}$ denote the intersection of all sets $\left(f_{\alpha}^{\beta}\right)^{-1}\left(Z_{\alpha} \times\{g(\alpha)\}\right)$ such that $0<\alpha \leqq \beta$. Then each $K_{\beta}^{g}$ is the intersection of all sets $\left(f_{\alpha}^{\beta}\right)^{-1}\left(K_{\alpha}^{g}\right)(\alpha \leqq \beta)$, while $K^{g}$ is the intersection of all $\left(f_{\beta}\right)^{-1}\left(K_{\beta}^{g}\right)(\beta<\tau)$. It follows by induction and the use of Lemma 2.6 that for each $\beta$ in $[0, \tau)$ and each $\alpha$ in $[0, \beta]$, $f_{\alpha}^{\beta} \mid K_{\beta}^{g}$ is a homeomorphism onto $K_{\alpha}^{g}$. Hence, again by Lemma 2.6, $f_{0} \mid K^{g}$ is a homeomorphism onto $Y$.

The proof of condition (5) is now immediate. For it follows from our construction and Lemma 2.2 that $w\left(Y_{\infty}\right) \leqq w(Y)$. Since $f_{0}$ is onto, $Y_{\infty}$ is nonempty; so by (4), $Y_{\infty}$ contains a copy of $Y$ as a subspace. Therefore, $w\left(Y_{\infty}\right)=w(Y)$.

Finally, to establish (6), let $\alpha \in[0, \tau)$ and suppose that $S$ is an $H(i)[R(i)]$ subspace of $Y_{\alpha}$. Let $y$ be a point of $\left(f_{\alpha}\right)^{-1}(S)$ and let $W$ be an open neighborhood of $y$ in $Y_{\infty}$. It follows from [4, 2.3(2), p. 428] and the way in which the system $\left\{Y_{\alpha} ; f_{\alpha}^{\beta}\right\}$ was constructed that for some $\beta$ in $[1, \tau), y_{\beta} \in V_{\beta} \times\{1,2\}$ and $\left(f_{\beta}\right)^{-1}\left(V_{\beta} \times\{1,2\}\right) \subset W$. Such a $\beta$ may be chosen so as to be greater than $\alpha$. Let $i$ be the member of $\{1,2\}$ such that $y_{\beta} \in V_{\beta} \times\{i\}$ and let $j$ denote the other member of $\{1,2\}$. Then $\left(f_{\beta}\right)^{-1}\left(V_{\beta} \times\{i\}\right)$ is an open set that contains $y$ and lies in $W$. To complete the proof it is sufficient to show that $\left(f_{\alpha}\right)^{-1}(S)-\left(f_{\beta}\right)^{-1}\left(V_{\beta} \times\{i\}\right)$ is $H(i)[R(i)]$. By (3), $f_{\alpha}$ is open, perfect and onto, which implies that $\phi_{\alpha}^{\beta}$ is open, perfect and onto. Since the restriction of $p_{\beta}$ to $Z_{\beta} \times\{j\}$ is a homeomorphism onto $Z_{\beta}$, and since $f_{\alpha}^{\beta}=\phi_{\alpha}^{\beta} p_{\beta}$, it follows that the restriction of $f_{\alpha}^{\beta}$ to $\left(\phi_{\alpha}^{\beta}\right)^{-1}(S) \times\{j\}$ is an open perfect mapping onto $S$. So by Lemma $2.7,\left(\phi_{\alpha}^{\beta}\right)^{-1}(S) \times\{j\}$ is $H(i)[R(i)]$. Therefore $\left(f_{\alpha}\right)^{-1}(S)-\left(f_{\beta}\right)^{-1}\left(V_{\beta} \times\{i\}\right)$, being the inverse of $\left(\phi_{\alpha}^{\beta}\right)^{-1}(S) \times\{j\}$ with respect to $f_{\beta}$, is also $H(i)$ [R(i)] (Lemma 2.7).

COROLlaRY 2.9. Every $H(i)$ space is the image, under an open perfect mapping, of a strongly $H(i i)$ space of the same weight. 
CoRollary 2.10. Every H-closed space is the image, under an open perfect mapping, of a $M H$ space of the same weight.

(In proving either Corollary 2.9 or Corollary 2.10 there are two cases to consider. For the case where the given space is of finite weight, and therefore compact, the proof is trivial. For the other case, the proof follows immediately from Theorem 2.8.)

COROLlaRY 2.11. Every nonempty $H(i)$ space is the image, under a perfect mapping, of a nonvacuously $H(i i)$ space.

Proof. Let $Y$ be a nonempty $H(i)$ space. Then by Corollary 2.9, there exist a strongly $H(i i)$ space $X$ and a perfect mapping $f$ of $X$ onto $Y$. Let $X^{\prime}$ be the space obtained by adding an extra isolated point to the space $X$. Then $X^{\prime}$ is a strongly $H(i i)$ space with an isolated point and is, therefore, nonvacuously $H(i i)$. To obtain a perfect mapping $g$ from $X^{\prime}$ onto $Y$, we simply choose $g$ to be a function from $X^{\prime}$ onto $Y$ such that $g \mid X=f$.

CoRollary 2.12. Every $H(i)$ [H-closed] space $Y$ can be expressed as the intersection of the members of a decreasing chain of strongly $H(i i)[M H]$ spaces, each of the same weight as $Y$.

Proof. If $w(Y)$ is finite then $Y$ is compact and the conclusion of the theorem is immediate. So suppose that $w(Y)$ is infinite and let $\tau$ be the least ordinal of cardinality $w(Y)$. Let $\left\{Y_{\alpha} ; f_{\alpha}^{\beta}\right\}$ be the inverse system guaranteed by Theorem 2.8, with $Y=Y_{0}$. By (3) and (4) of 2.8, there is a subspace $K$ of $Y_{\infty}$ such that $f_{0} \mid K$ is a homeomorphism of $K$ onto $Y$. Now for each $\alpha$ in $[0, \tau)$, let $X_{\alpha}=\left(f_{\alpha}\right)^{-1} f_{\alpha}(K)$. Then $\left\{X_{\alpha} \mid \alpha \in[0, \tau)\right\}$ is a decreasing chain and $K$ is the intersection all $X_{\alpha}$ 's $(\alpha<\tau)$. It follows from 2.8(6) that for each $\alpha$ in $[0, \tau), X_{\alpha}$ is strongly $H(i i)[M H]$. Finally, since each of $K$ and $Y_{\infty}$ is of weight $w(Y)(2.8(5))$ and since each $X_{\alpha}$ contains $K$ and lies in $Y_{\infty}$, we conclude that each $X_{\alpha}$ is of weight $w(Y)$. So by identifying $Y$ with $K$, we obtain the desired conclusion.

For Corollaries 2.13 through 2.16, which follow, the proofs are analogous to those of 2.9 through 2.12 , respectively.

COROLlaRY 2.13. Every $R(i)[R(i)$ Hausdorff] space is the image, under an open perfect mapping, of a strongly $R(i i)$ [strongly $R(i i)$ Hausdorff] space of the same weight.

COROLlaRY 2.14. Every $R$-closed space is the image, under an 
open perfect mapping, of a SMR space of the same weight.

COROLlaRY 2.15. Every nonempty $R(i)$ [R(i) Hausdorff] space is the image, under a perfect mapping, of a nonvacuously $R(i i)$ [nonvacuously $R(i i)$ Hausdorff] space.

CoRollaRY 2.16. Every $R(i)$ [ $R(i)$ Hausdorff; $R$-closed] space $Y$ can be expressed as the intersection of the members of a decreasing chain of strongly $R(i i)$ [strongly $R(i i)$ Hausdorff; SMR] spaces, each of the same weight as $Y$.

It is not known if a product of $R$-closed spaces is always $R$ closed or if a product of $M R$ spaces is always $M R$. The following theorem shows that an affirmative answer to the second question would imply an affirmative answer to the first.

THEOREM 2.17. Let af be a set. If every collection of SMR spaces indexed over $\mathscr{A}$ yields an $R$-closed product, then every collection of $R$-closed spaces indexed over $\mathscr{A}$ yields an $R$-closed product.

Proof. Let $\left\{Y_{\alpha} \mid \alpha \in \mathscr{A}\right\}$ be a collection of $R$-closed spaces. By Corollary 2.14, there exists a collection $\left\{\left(X_{\alpha}, f_{\alpha}\right) \mid \alpha \in \mathscr{A}\right\}$ such that for each $\alpha$ in $\mathscr{A}, X_{\alpha}$ is an $S M R$ space and $f_{\alpha}$ is a continuous function from $X_{\alpha}$ onto $Y_{\alpha}$. Then $\Pi X_{\alpha}(\alpha \in \mathscr{A})$ is $R$-closed. Since the collection $\left\{f_{\alpha} \mid \alpha \in \mathscr{A}\right\}$ induces a continuous function from $\Pi X_{\alpha}$ onto $\Pi Y_{\alpha}$, this implies that $\Pi Y_{\alpha}$ is $R$-closed.

In [12] Stephenson gave an example of an $R(i)$ Hausdorff space whose product with itself is not $R(i)$. Using Stephenson's example, Corollary 2.15, and the method used in the proof of Theorem 2.17, we obtain an example which answers a question of Scarborough and Stone [10, p. 138] concerning $R(i i)$ spaces.

EXAMPLE 2.18. A nonvacuously $R(i i)$ Hausdorff space whose product with itself is neither $R(i)$ nor $R(i i)$.

Let $Y$ be the space described by Stephenson in [12]. By Corollary 2.15 , there exists a nonvacuously $R(i i)$ Hausdorff space $X$ and a continuous function $f$ from $X$ onto $Y$. Since $f$ induces a continuous function from $X \times X$ onto $Y \times Y$, we conclude that $X \times X$ is not $R(i)$. But because $X$ is nonvacuously $R(i i), X \times X$ will necessarily have a point which is the unique cluster point of a regular filterbase on $X \times X$. Since a nonvacuously $R(i i)$ space is always $R(i)$, this implies that $X \times X$ is not $R(i i)$. 
A topological space is said to be e-compact with respect to a dense subset $D$ if every ultrafilter on $D$ converges to a point of $X$. A space which is $e$-compact with respect to some dense subset is called an e-compact space. (There definitions are due to S. Hechler [7].)

In [7] Hechler described a non-e-compact $H$-closed space. Stephenson has recently produced an example of a non-e-compact $M H$ space (see [11]). Corollary 2.9 provides us with another method of obtaining such an example.

\section{EXAmPLe 2.19. A non-e-compact $M H$ space.}

Let $Y$ be a non-e-compact $H$-closed space. (See, for example [7, Corollary 3.2, p. 223].) By Corollary 2.9, there is an $M H$ space $X$ which can be mapped continuously onto $Y$. Since the continuous image of an $e$-compact space is e-compact [7, Theorem 2.3, p. 220], the space $X$ is not $e$-compact.

3. Inverse limits. Since inverse limits were shown in $\S 2$ to be useful in constructing pre-images of $H$-closed and $R$-closed spaces, it seems natural to ask about the preservation of minimal properties by inverse limits. As Example 3.1 below shows, the inverse limit of $H$-closed spaces need not be $H$-closed even if each bonding map is an open embedding and the system is indexed over the natural numbers. We can, however, obtain some partial results.

EXAMPLE 3.1. An inverse system of $M H$ spaces such that the bounding maps are open embeddings but the inverse limit is not $H$-closed.

We shall construct a nested sequence of $M H$ spaces such that each inclusion map is open and the intersection of the spaces is not $H$-closed. Let $Y_{1}$ denote the noncompact $M H$ space described in [2] as Example 3.14. Let

$$
\begin{gathered}
Y_{2}=Y_{1}-\left\{a_{11}, b_{11}\right\}, Y_{3}=Y_{2}-\left\{a_{12}, b_{12}, a_{21}, b_{21}\right\}, \cdots, \\
Y_{n+1}=Y_{n}-\left\{a_{i j}, b_{i j} \mid i+j=n+1\right\}, \cdots .
\end{gathered}
$$

For each $n$ in $N, Y_{n+1}$ is an open $M H$ subspace of $Y_{n}$. But $\bigcap_{n=1}^{\infty} Y_{n}$ is the infinite subspace $\{a\} \cup\{b\} \cup\left\{c_{2}\right\}_{i=1}^{\infty}$ of $Y_{1}$, and since this subspace has the discrete topology it is not $H$-closed.

REMARK. In a similar manner it is possible to construct an example of a nested sequence of $R$-closed spaces with a non- $R$-closed intersection, showing that the inverse limit of $R$-closed spaces need not be $R$-closed. The noncompact $R$-closed space of [2, Example 
4.19] can be used as the first space of the sequence.

Definition 3.2. A continuous function $f: X \rightarrow Y$ is said to have the inverse property if $\mathrm{Cl} f^{-1}(U)=f^{-1}(\bar{U})$ for every open set $U$ in $Y$.

THEOREM 3.3. If $\left\{X_{\alpha} ; f_{\alpha}^{\beta}\right\}$ is an inverse system of H-closed spaces such that each projection $f_{\alpha}$ has the inverse property, then $X_{\infty}$ is $H$-closed.

Proof. Since by Zorn's lemma every open filterbase is contained in a maximal open filter (i.e., an open filterbase which is not properly contained in an open filterbase), it is sufficient to show that every maximal open filter on $X_{\infty}$ converges. So let $\mathscr{U}$ be a maximal open filter on $X_{\infty}$. Let $\gamma$ be an element of the index set for the system and let $\left(f_{r}\right)^{\sharp}(\mathscr{C})=\left\{V\right.$ open in $X_{r} \mid$ for some $U$ in $\left.\mathscr{L}, f_{r}(U) \subseteq V\right\}$. Then $\left(f_{r}\right)^{\sharp}\left(\mathscr{C}_{C}\right)$ is an open filterbase in $X_{r}$ and, since $X_{r}$ is $H$-closed, has a cluster point $x_{r}$ in $X_{r}$. Let $W$ be an open neighborhood of $x_{r}$ in $X_{\gamma}$. We assert that for each $U$ in $\mathscr{Q},\left(f_{\gamma}\right)^{-1}(W) \cap U$ is nonempty. For assume that $U_{0} \in \mathscr{U}$ and $\left(f_{r}\right)^{-1}(W) \cap U_{0}=\varnothing$. Since $U_{0}$ is open, this implies that $\mathrm{Cl}\left(f_{\gamma}\right)^{-1}(W) \cap U_{0}=\varnothing$ and therefore, since $f_{\gamma}$ has the inverse property, that $\left(f_{r}\right)^{-1}(\bar{W}) \cap U_{0}=\varnothing$. Then

$$
U_{0} \subseteq\left(f_{r}\right)^{-1}\left(X_{r}-\bar{W}\right),
$$

from which it follows that $\left(X_{\gamma}-\bar{W}\right) \in\left(f_{\gamma}\right)^{\sharp}(\mathscr{C})$. Since $x_{\gamma} \in W$ and $x_{\gamma}$ is a cluster point of $\left(f_{r}\right)^{*}(\mathscr{C})$, this is impossible. Hence, $\left(f_{r}\right)^{-1}(W)$ intersects each member of $\mathscr{U}$. But this implies, since $\mathscr{U}$ is a maximal open filter on $X_{\infty}$, that $\left(f_{r}\right)^{-1}(W) \in \mathscr{U}$, and that $W \in\left(f_{r}\right)^{\sharp}(\mathscr{U})$. Therefore $f_{\gamma}\left(\mathscr{U}_{L}\right)$ converges to $x_{\gamma}$. For each $\alpha$, then, we can find an $x_{\alpha}$ in $X_{\alpha}$ such that $f_{\alpha}(\mathscr{U})$ converges to $x_{\alpha}$. We now claim that $\left\{x_{\alpha}\right\}$ is in $X_{\infty}$. Suppose $\nu$ and $\beta$ are members of the index set and that $\nu<\beta$. Then $f_{\beta}(\mathscr{U})$ converges to $x_{\beta}$ and $f_{\nu}^{\beta} f_{\beta}(\mathscr{U})$ converges to $f_{\nu}^{\beta}\left(x_{\beta}\right)$. But $f_{\nu}^{\beta} f_{\beta}=f_{\nu}$ and $f_{\nu}(\mathscr{U})$ converges to $x_{\nu}$; hence, $f_{\nu}^{\beta}\left(x_{\beta}\right)=x_{\nu}$. So we conclude that $\left\{x_{\alpha}\right\}$ is in $X_{\infty}$. It now follows from [5, 3.2(2), p. 428] that $\mathscr{U}$ converges to $\left\{x_{\alpha}\right\}$. This completes the proof.

THeOREM 3.4. If $\left\{X_{\alpha} ; f_{\alpha}^{\beta}\right\}$ is an inverse system of $M H$ spaces such that each projection $f_{\alpha}$ has the inverse property, then $X_{\infty}$ is MH.

Proof. Let $\mathscr{W}$ be an open filterbase on $X_{\infty}$ such that $\mathscr{U}$ has a unique cluster point $x$. For each $\alpha$, let $\left(f_{\alpha}\right)^{\sharp}(\mathscr{U})=\left\{V\right.$ open in $X_{\alpha} \mid$ for some $U$ in $\left.\mathscr{U}, f_{\alpha}(U) \subseteq V\right\}$. Then for each $\alpha$ we have $\left(f_{\alpha}\right)^{\sharp}(\mathscr{U})$ an open filterbase, $f_{\alpha}(\mathscr{U})$ a filterbase subordinate to $\left(f_{\alpha}\right)^{\sharp}(\mathscr{U})$, and $x_{\alpha}$ a cluster point of $f_{\alpha}(\mathscr{U})$; hence $x_{\alpha}$ is a cluster point of $\left(f_{\alpha}\right)^{\sharp}(\mathscr{U})$. Now 
suppose that $\gamma$ is an element of the index set and that $p$ is a cluster point of $\left(f_{\gamma}\right)^{\sharp}(\mathscr{U})$. For every open neighborhood $W$ of $p$ and every $U$ in $\mathscr{Q}$ we have, as in the proof of $3.3,\left(f_{r}\right)^{-1}(W) \cap U \neq \varnothing$. Since $X_{r}$ is $H$-closed (Theorem 3.3) the open filterbase $\left\{\left(f_{r}\right)^{-1}(W) \cap U \mid W\right.$ an open neighborhood of $p, U \in \mathscr{C}\}$ must have a cluster point $y$ in $X_{\infty}$. Clearly, $y_{r}=p$. But $y$ is also a cluster point of $\mathscr{U}$, implying that $y=x$ and $p=x_{\gamma}$. So for each $\alpha, x_{\alpha}$ is the only cluster point of $\left(f_{\alpha}\right)^{\sharp}(\mathscr{C})$ and, therefore, $\left(f_{\alpha}\right)^{\sharp}(\mathscr{U})$ converges to $x_{\alpha}$. From this it follows that $f_{\alpha}(\mathscr{U})$ converges to $x_{\alpha}$ for each $\alpha$ and, consequently, that $\mathscr{U}$ converges to $x$. Hence, $X_{\infty}$ is $M H$.

THEOREM 3.5. If $\left\{X_{\alpha} ; f_{\alpha}^{\beta}\right\}$ is an inverse system of nonempty $H$ closed spaces, indexed over a directed set having a cofinal simple chain, then $X_{\infty}$ is nonempty.

Proof. Since the directed set over which $\left\{X_{\alpha} ; f_{\alpha}^{\beta}\right\}$ is indexed has a cofinal simple chain and since every simply ordered set has a cofinal well-ordered chain, we may assume that for some nonzero ordinal $\tau,\left\{X_{\alpha} ; f_{\alpha}^{\beta}\right\}$ is indexed over $[0, \tau)$ (see [5, 2.7, p. 431]). For each $\beta$ in $[0, \tau)$, let $H_{\beta}^{\prime}=\lim X_{\alpha}(\alpha \leqq \beta)$ and let $H_{\beta}=H_{\beta}^{\prime} \times \Pi X_{\alpha}$ $(\alpha>\beta)$. Then for each $\beta, \overleftarrow{H_{\beta}^{\prime}}$ is homeomorphic to $X_{\beta}$ and, consequently, $H_{\beta}$ is $H$-closed and nonempty [2,3.3(c)]. It is easily verified that $\left\{H_{\beta} \mid \beta<\tau\right\}$ is a nonempty decreasing chain of subsets of $\Pi X_{\alpha}$. Hence, $\bigcap H_{\beta}(\beta<\tau)$ is nonempty [2,3.3(e)]. Since a point of $\bigcap H_{\beta}$ will necessarily be in $X_{\infty}$, this completes the proof.

THeOREM 3.6. Let $\left\{X_{\alpha} ; f_{\alpha}^{\beta}\right\}$ be an inverse system of H-closed [R-closed] spaces indexed over a directed set having a cofinal simple chain. If each bonding map $f_{\alpha}^{\beta}$ is onto then each projection $f_{\alpha}$ is onto.

Proof. As in the proof of 3.5, we may assume that for some nonzero ordinal $\tau$ the system is indexed over $[0, \tau)$. Let $\beta$ be an ordinal in $[0, \tau)$ and let $p$ be a point of $X_{\beta}$. We shall identify a point $\left\{y_{\alpha}\right\}$ of $X_{\infty}$ such that $y_{\beta}=p$. For $\alpha$ in $[0, \beta]$, let $y_{\alpha}=f_{\alpha}^{\beta}(p)$. The remaining coordinates of $\left\{y_{\alpha}\right\}$ are chosen inductively as follows. Suppose that for each $\alpha$ in $[0, \gamma), y_{\alpha}$ has already been chosen. For each $\alpha$ in $[0, \gamma)$, choose a neighborhood base $\mathscr{B}_{\alpha}$ at $y_{\alpha}$ and let $\mathscr{F}_{\alpha}=\left\{\left(f_{\alpha}^{\gamma}\right)^{-1}(V) \mid V \in \mathscr{B}_{\alpha}\right\}$. Let $\mathscr{F}=\bigcup \mathscr{F}_{\alpha}(\alpha<\gamma)$. Then, since each bonding map is onto, $\mathscr{F}$ is an open [regular] filterbase on $X_{\gamma}$ and must have a cluster point in $X_{r}$. Now, for each $\alpha$ in $[0, \gamma)$, we have $\mathscr{B}_{\alpha} \subseteq\left\{f_{\alpha}^{\gamma}(W) \mid W \in \mathscr{F}\right\}$, which implies that $y_{\alpha}$ is the only cluster point of $\left\{f_{\alpha}^{\gamma}(W) \mid W \in \mathscr{F}\right\}$ in $X_{\alpha}$. Hence, if we choose $y_{\gamma}$ to be a cluster point of $\mathscr{F}$ in $X_{\gamma}$, then for each $\alpha$ in $[0, \gamma)$ we have $f_{\alpha}^{\gamma}\left(y_{\gamma}\right)=y_{\alpha}$. 
LEMMA 3.7. If $\left\{X_{\alpha} ; f_{\alpha}^{\beta}\right\}$ is an inverse system such that each projection $f_{\alpha}: X_{\infty} \rightarrow X_{\alpha}$ is onto and each bonding map $f_{\alpha}^{\beta}: X_{\beta} \rightarrow X_{\alpha}$ has the inverse property, then each projection has the inverse property.

Proof. Let $U$ be open in $X_{\alpha}$. Let $y$ be a point of $\left(f_{\alpha}\right)^{-1}(\bar{U})$ and $W$ an open neighborhood of $y$ in $X_{\infty}$. Then there is a $\beta$ greater than or equal to $\alpha$ and an open set $V$ in $X_{\beta}$ such that $y_{\beta} \in V$ and $\left(f_{\beta}\right)^{-1}(V) \leqq W$. Since $\left(f_{\alpha}^{\beta}\right)^{-1}(\bar{U})=\mathrm{Cl}\left(f_{\alpha}^{\beta}\right)^{-1}(U)$ we have $y_{\beta} \in \mathrm{Cl}\left(f_{\alpha}^{\beta}\right)^{-1}(U)$ and, therefore, $V \cap\left(f_{\alpha}^{\beta}\right)^{-1}(U) \neq \varnothing$. Hence,

$$
W \cap\left(f_{\alpha}\right)^{-1}(U) \neq \varnothing,
$$

and we conclude that $y \in \mathrm{Cl}\left(f_{\alpha}\right)^{-1}(U)$. Since the continuity of $f_{\alpha}$ implies that $\mathrm{Cl}\left(f_{\alpha}\right)^{-1}(U) \subseteq\left(f_{\alpha}\right)^{-1}(\bar{U})$, this establishes the equality of $\mathrm{Cl}\left(f_{a}\right)^{-1}(U)$ and $\left(f_{\alpha}\right)^{-1}(\bar{U})$.

THEOREM 3.8. Let $\left\{X_{\alpha} ; f_{\alpha}^{\beta}\right\}$ be an inverse system of $H$-closed $[M H]$ spaces indexed over a directed set having a cofinal simple chain. If each bonding map $f_{\alpha}^{\beta}$ is onto and has the inverse property then $X_{\infty}$ is $H$-closed [MH].

Theorem 3.8 follows immediately from 3.6, 3.7, 3.3 and 3.4. Since every open mapping has the inverse property, we also have the following corollary.

CoROLlaRY 3.9. Let $\left\{X_{\alpha} ; f_{\alpha}^{\beta}\right\}$ be an inverse system of $H$-closed $[M H]$ spaces indexed over a directed set having a cofinal simple chain. If each bonding map $f_{\alpha}^{\beta}$ is onto and open then $X_{\infty}$ is $H$ closed $[M H]$.

REMARK. Example 3.1 shows that the requirement that each bonding map be onto cannot be deleted from the hypothesis of Theorem 3.8 (or of Corollary 3.9).

QUESTIONS 3.10.

(1) Is there a theorem analogous to 3.5 for $R$-closed spaces?

(2) Can the requirement that the index set have a cofinal simple chain be removed from $3.5,3.6,3.8$ or 3.9 ?

Remarks. A mapping $f$ from a space $X$ to a space $Y$ is called a $p$-map if for each open cover $\mathscr{V}$ of $Y$ containing a finite proximate subcover of $Y, f^{-1}(\mathscr{V})$ contains a finite proximate subcover of $X$. (Clearly, every mapping with the inverse property is a $p$-map.) It follows from [6, Theorem B] and [8, Theorem 3.7] that the 
inverse limit of a system of $H$-closed spaces is $H$-closed if all bonding maps and projections are p-maps. This result, in combination with 3.6 and 3.7 , yields an alternate proof of the $H$-closed case of Theorem 3.8 .

The authors are grateful to Professor George Strecker for bringing paper [6] to their attention.

4. Finite-to-one mappings. Although Corollary 2.10 more than answers the original question raised by Willard, the method used in obtaining 2.10 yields pre-images and mappings which are quite complicated. In general, point inverses are very large, even when the given $H$-closed space has only finitely many bad points. Since many of the standard examples on noncompact $H$-closed spaces (such as [2, Example 3.13]) are finite-to-one continuous images of $M H$ spaces, it would seem desirable to have a general method which, for certain classes of $H$-closed spaces, would give simpler pre-images and mappings than those obtained in $\S 2$. In this section we show (Theorem 4.2) that if the given $H$-closed space has only finitely many bad points and if each bad point has a countable neighborhood base, then there is an $M H$ space that is taken onto the given space by an at-most-two-to-one mapping having the inverse property defined in $\S 3$.

Example 4.5 shows that Theorem 4.2 cannot be strengthened so as to guarantee that the mapping will be open (or even a quotient mapping), while Example 4.3 shows that the countable neighborhood base requirement cannot be removed from the hypothesis. Example 4.6 shows that we cannot hope for a result analogous to 4.2 for $R$ closed and $M R$ spaces.

Lemma 4.1. Let $Y$ be an H-closed space, $y^{*}$ a bad point of $Y$ having a countable neighborhood base, and $V$ a neighborhood of $y^{*}$. Then there exists an H-closed space $X$ and an at-most-two-to-one mapping $f$ of $X$ onto $Y$ such that (1) $f$ has the inverse property, (2) the restriction of $f$ to $f^{-1}(Y-V)$ is a homeomorphism, and (3) $X$ is semiregular at each point $x$ for which either $f(x)$ is semiregular in $Y$ or $f(x)=y^{*}$.

Proof. We may assume that $V$ contains no regularly open neighborhood of $y^{*}$ and that $V$ is the first member of a countable nested neighborhood base $\left\{U_{n}\right\}_{n=1}^{\infty}$ at $y^{*}$. Let $F=\bigcup_{n=1}^{\infty}\left(\mathrm{Cl}_{Y} U_{n}-U_{n}\right)$. It is easily verified that for each $n$ in $N, F \cup \mathrm{Cl}_{Y} U_{n}$ is closed. Since $Y$ is Hausdorff we have $F \cup\left\{y^{*}\right\}=\bigcap_{n=1}^{\infty}\left(F \cup \mathrm{Cl}_{Y} U_{n}\right)$, and therefore $F \cup\left\{y^{*}\right\}$ is closed in $Y$. Let $\mathscr{T}$ be the topology of $Y$, and for each $U$ in $\mathscr{T}$ let $U^{\prime}=U-F$. Let $Y^{\prime}$ denote the space whose points are the points of $Y$ and whose topology is generated by $\mathscr{T} \cup\left\{U^{\prime} \mid U \in \mathscr{T}\right\}$. 
The topology of $Y^{\prime}$ will be denoted as $\mathscr{T}^{\prime}$. It readily follows that $\left\{U_{n}^{\prime}\right\}_{n=1}^{\infty}$ is a neighborhood base for $y^{*}$ in $Y^{\prime}$.

We assert that if $U \in \mathscr{T}$, then $\mathrm{Cl}_{Y^{\prime}} U^{\prime}=\mathrm{Cl}_{Y}, U=\mathrm{Cl}_{Y} U$. Since we clearly have $\mathrm{Cl}_{Y}, U^{\prime} \subseteq \mathrm{Cl}_{Y^{\prime}} U \subseteq \mathrm{Cl}_{Y} U$, we have only to show that $\mathrm{Cl}_{Y} U \subseteq \mathrm{Cl}_{Y^{\prime}} U^{\prime}$. Let $y$ be a point of $\mathrm{Cl}_{Y} U$ and $W$ a $\mathscr{T}$-open neighborhood of $y$. It is sufficient to show that $W^{\prime} \cap U^{\prime} \neq \varnothing$. Since $y \in \mathrm{Cl}_{Y} U$, there is a point $p$ of $U$ in $W$. If $p \notin F$ then $p \in W^{\prime} \cap U^{\prime}$ and we are finished. So suppose that $p \in F$. Since $y^{*}$ is not in $F$ (and therefore not equal to $p$ ), there exists a $k$ in $N$ such that $p \in \mathrm{Cl}_{Y} U_{k}-U_{k}$ and $p \notin \mathrm{Cl}_{Y} U_{k+1}-U_{k+1}$. Because $U_{k+1} \cong U_{k}$, this means that $p \notin \mathrm{Cl}_{Y} U_{k+1}$. Therefore, $(W \cap U)-\mathrm{Cl}_{Y} U_{k+1}$ is a $\mathscr{T}$-open neighborhood of $p$ and must contain a point $z$ of $U_{k}$. But then $z \in U_{k}-\mathrm{Cl}_{Y} U_{k+1}$, and since $U_{k}-\mathrm{Cl}_{Y} U_{k+1}$ is a subset of $U_{k}^{\prime}$ this implies that $z \notin F$. Hence, we have $W^{\prime} \cap U^{\prime} \neq \varnothing$, and our assertion is proved.

We next assert that $Y^{\prime}$ is $H$-closed. For let $\mathscr{G}$ be a $\mathscr{T}^{\prime}$-open cover of $Y^{\prime}$. We may assume that each member of $\mathscr{G}$ is in $\mathscr{T} \cup\left\{U^{\prime} \mid U \in \mathscr{T}\right\}$. Let $\mathscr{C}$ denote the collection $\{U \in \mathscr{T} \mid U \in \mathscr{G}$ or $\left.U^{\prime} \in \mathscr{G}\right\}$. Then $\mathscr{C}$ is a $\mathscr{T}$-open cover of $Y$ and has a finite subcollection which (with respect to $\mathscr{T}$ ) is a proximate cover of $Y$. Since for each $U$ in $\mathscr{C}, \mathrm{Cl}_{Y} U=\mathrm{Cl}_{Y^{\prime}} U=\mathrm{Cl}_{Y^{\prime}} U^{\prime}$, it follows that $\mathscr{G}$ has a finite subcollection which (with respect to $\mathscr{T}^{\prime}$ ) is a proximate cover of $Y^{\prime}$. Hence, by [2,3.2], the assertion is verified.

Now let $X$ be the quotient space obtained from $Y^{\prime} \times\{1,2\}$ by identifying $(y, 1)$ with $(y, 2)$ for each $y$ in $Y^{\prime}-V^{\prime}$. Since $X$ is the union of two $H$-closed subsets of itself $\left(Y^{\prime} \times\{1\}\right.$ and $\left.Y^{\prime} \times\{2\}\right), X$ is also $H$-closed. Let $f$ be the natural projection from $X$ onto $Y$ (i.e., for each $(y, i)$ in $\left.Y^{\prime} \times\{1,2\}, f(y, i)=y\right)$. Then $f$ is a continuous function from $X$ onto $Y$. Since for each $U$ in $\mathscr{C}, \mathrm{Cl}_{Y} U=\mathrm{Cl}_{Y^{\prime}} U$, it follows that $f$ has the inverse property.

We wish to show that $X$ is semiregular at each point of $f^{-1}\left(y^{*}\right)$. For each $(n, i)$ in $N \times\{1,2\}$, let $U_{n i}^{\prime}=U_{n}^{\prime} \times\{i\}$. Clearly $\left\{U_{n 1}^{\prime}\right\}_{n=1}^{\infty}$ is a neighborhood base for $\left(y^{*}, 1\right)$ in $X$. Let $k \in N$ and let $q \in \mathrm{Cl}_{X} U_{k_{1}}^{\prime}$ $U_{k 1}^{\prime}$. Then $f(q) \in \mathrm{Cl}_{Y^{\prime}}, U_{k}^{\prime}-U_{k}^{\prime}$, and since

$$
\mathrm{Cl}_{Y^{\prime}} U_{k}^{\prime}-U_{k}^{\prime} \cong F \cong Y^{\prime}-V^{\prime},
$$

this implies that $q \in \mathrm{Cl}_{X} U_{k 2}^{\prime}$. But since $U_{k}^{\prime} \subseteq V^{\prime}$, we have $U_{k_{1}}^{\prime}$ and $U_{k 2}^{\prime}$ disjoint open sets in $X$; therefore, $q \notin \operatorname{int}_{X} \mathrm{Cl}_{X} U_{k 1}^{\prime}$. We conclude, then, that for each $n$, int $\operatorname{Cl}_{X}{\mathrm{Cl}_{n}}_{n 1}^{\prime}=U_{n 1}^{\prime}$; i.e., the members of $\left\{U_{n 1}^{\prime}\right\}_{n=1}^{\infty}$ are regularly open in $X$. Hence, $\left(y^{*}, 1\right)$ is a semiregular point of $x$. By analogous argument, $\left(y^{*}, 2\right)$ is also a semiregular point of $X$.

To complete the proof of the lemma, let $x$ be a point of $X$ such that $Y$ is semiregular at $f(x)$. Since $F \cup\left\{y^{*}\right\}$ is closed in $Y$, each 
member of $\mathscr{T}^{\prime}$ which misses $y^{*}$ is also a member of $\mathscr{T}$. Hence, the subspace $Y-\left\{y^{*}\right\}$ of $Y$ has the same topology as the subspace $Y^{\prime}-\left\{y^{*}\right\}$ of $Y^{\prime}$. Since $f(x)$ is in $Y-\left\{y^{*}\right\}$, this implies that $Y^{\prime}$ is semiregular at $f(x)$. But $f$ is an open mapping from $X$ onto $Y^{\prime}$, so it now follows that $X$ is semiregular at $x$.

TheOREM 4.2. If $Y$ is an H-closed space having only finitely many bad points, and if at each of these bad points there is a countable neighborhood base, then $Y$ is the at-most-two-to-one image of an $M H$ space under a mapping having the inverse property.

Proof. Since $Y$ is Hausdorff, we may separate the finitely many bad points by disjoint open sets. The desired result is then obtained by applying Lemma 4.1 finitely many times.

ExAMPle 4.3. An $H$-closed space that has only one bad point but is not the finite-to-one continuous image of an $M H$ space.

Let $I$ denote the closed real-line interval from 0 to 1 and $\mathscr{Q}$ the usual topology on $I$. Let $\mathscr{H}$ be the collection of all countable infinite subsets $H$ of $I-\{0\}$ such that, with respect to $\mathscr{C}, 0$ is the only limit point of $H$. Now $\{U-H \mid U \in \mathscr{U}, H \in \mathscr{H}\}$ is a base for a topology $\mathscr{T}$ on $I$. We shall let $Y$ denote the space $(I, \mathscr{T})$. Since $\mathscr{U} \subseteq \mathscr{T}, Y$ is Hausdorff. Since every member of $\mathscr{T}$ that lies in $I-\{0\}$ is also a member of $\mathscr{K}, Y$ is semiregular at every point of $I-\{0\}$. And for each $V$ in $\mathscr{T}$ there is a $U$ in $\mathscr{Q}$ such that $V \subseteq U$ and $\mathrm{Cl}_{\mathscr{T}} V=\mathrm{Cl}_{\mathscr{L}} U$. Hence, it follows from the compactness of $(I, \mathscr{U})$ that every open cover of $Y$ has a finite subcollection which is a proximate cover of $Y$; i.e., $Y$ is $H$-closed.

Assume that for some $M H$ space $X$ there is a finite-to-one mapping $f$ from $X$ onto $Y$. For each $n$ in $N$, let $B_{n}$ denote the inverse (with respect to $f$ ) of the open interval $(0,1 / n)$ in $I$. Then $\left\{B_{n} \mid n \in N\right\}$ is an open filterbase on $X$ and must have a cluster point $p$ in $X$. Since $f^{-1}(0)$ is finite and $X$ is Hausdorff, we can choose an open neighborhood $W$ of $p$ such that $f^{-1}(0)-\{p\} \subseteq X-\bar{W}$. Clearly, every cluster point of $\left\{B_{n} \mid n \in N\right\}$ is in $f^{-1}(0)$, so $p$ is the only cluster point of $\left\{B_{n} \mid n \in N\right\}$ in $\bar{W}$. Then $\left\{B_{n} \cap W \mid n \in N\right\}$ is an open filterbase with unique cluster point $p$ and must therefore converge to $p$. For each $n$ in $N$, choose a point $x_{n}$ of $B_{n} \cap W$. Then $\left\{f\left(x_{n}\right) \mid n \in N\right\}$ is a member of $\mathscr{H}$, which implies that $W-\bigcup_{n=1}^{\infty} f^{-1} f\left(x_{n}\right)$ is an open neighborhood of $p$. But since no member of $\left\{B_{n} \cap W \mid n \in N\right\}$ is in $W-\bigcup_{n=1}^{\infty} f^{-1} f\left(x_{n}\right)$, we have a contradiction. Hence, we conclude that $Y$ is not the finite-to-one continuous image of an $M H$ space. 
QUestion 4.4. Is every first countable $H$-closed space the finiteto-one continuous image of an $M H$ space?

Conjecture. Let $I^{2}$ denote the unit square in the plane, bd $I^{2}$ the boundary of $I^{2}$ in the plane, and of the usual relative topology on $I^{2}$. Let $Y$ be the first countable $H$-closed space obtained by giving to $I^{2}$ the topology $\left\{V-F \mid V \in \mathscr{Y}, F \subseteq\right.$ bd $\left.I^{2}\right\}$. Then $Y$ is not the image, under a finite-to-one mapping, of an $M H$ space.

The following example shows that Theorem 4.2 cannot be strengthened so as to guarantee that the given $H$-closed space be the finite-to-one image of an $M H$ space under a quotient mapping.

EXAMPLE 4.5. A first countable $H$-closed space that has only one bad point but is not the finite-to-one image of an $M H$ space under a quotient mapping.

Let $I$ denote the closed real-line interval from 0 to 1 and $\mathscr{W}$ the usual topology on $I$. For each $m$ in $N$, let $A_{m}=\{(1 / 2) n(n+1)+i \mid n \in N$, $i \in N \cup\{0\}, 0 \leqq i \leqq m\}$ and let $C_{m}=\left\{1 / a \mid a \in A_{m}\right\}$. Then for every $m$ in $N, C_{m+1}-C_{m}$ is the range of an infinite sequence in $I-\{0\}$ which (with respect to $\mathscr{C}$ ) converges to 0 . The collection

$$
\mathscr{L} \cup\left\{U-C_{m} \mid U \in \mathscr{K}, m \in N\right\}
$$

is a base for a topology $\mathscr{T}$ on $I$. The topological space $(I, \mathscr{T})$, which we shall denote as $Y$, is first countable and $H$-closed and has only 0 as a bad point.

Assume that for some $M H$ space $X$ there is a finite-to-one quotient mapping $f$ of $X$ onto $Y$. Let $\mathscr{U}_{0}=\{U \in \mathscr{U} \mid 0 \in U\}$. Since $f^{-1}(0)$ is finite and $X$ is Hausdorff, we can choose for each $p$ in $f^{-1}(0)$ an open set $W_{p}$ in $X$ such that $p \in W_{p}$ and $\bar{W}_{p} \cap f^{-1}(0)=\{p\}$. Let $W$ denote the union of these $W_{p}$ 's. Now because $f$ is a quotient mapping and $Y$ is first countable, it follows that $f$ is pseudo-open; i.e., for each $y$ in $Y$, every neighborhood of $f^{-1}(y)$ in $X$ is taken by $f$ onto a neighborhood of $y$ in $Y$ (see [1, Theorem 4]). Thus, for some $V$ in $\mathscr{U}_{0}$ and some $j$ in $N, V-C_{j} \subseteq f(W)$. Since $C_{j+1}-C_{j}$ is infinite and $f^{-1}(0)$ is finite, there is some $q$ in $f^{-1}(0)$ such that $W_{q} \cap$ $f^{-1}\left(C_{j+1}\right)$ is infinite; and since every member of $\mathscr{L}_{0}$ contains all but finitely many members of $C_{j+1}$ we have, for each $U$ in $\mathscr{U}_{0}$,

$$
W_{q} \cap f^{-1}(U) \cap f^{-1}\left(C_{j+1}\right) \neq \varnothing .
$$

Now let $\mathscr{B}=\left\{W_{q} \cap f^{-1}(U) \mid U \in \mathscr{U}_{0}\right\}$. Clearly, $W_{q}-f^{-1}\left(C_{j+1}\right)$ is an open neighborhood of $q$ and contains no member of the open filterbase $\mathscr{B}$. Since $X$ is $M H, \mathscr{B}$ must have a cluster point distinct from $q$. 
But such a cluster point must lie in $f^{-1}(0)$ and also in $\bar{W}_{q}$. This contradicts the fact that $\bar{W}_{q} \cap f^{-1}(0)=\{q\}$.

EXAMPLE 4.6. An $R$-closed space that has a countable neighborhood base at its only bad point but is not the finite-to-one continuous image of an $M R$ space.

Let $S$ be an $R$-closed non-MR space which has only one bad point and which has a countable neighborhood base at that bad point (such as the space of $[2,4.19]$ ). Let $S_{1}, S_{2}, S_{3}, \cdots$ be disjoint copies of $S$ with $p_{1}, p_{2}, p_{3}, \cdots$ the respective bad points. Now for each $n$ in $N$ identify $p_{n}$ with $p_{1}$, and let $p^{*}$ denote this point. Define a topological space $Y$ as follows. The points of $Y$ will be the points of $\bigcup_{n=1}^{\infty} S_{n}$ (with the $p_{n}$ 's identified). A set $V$ in the space will be open if and only if (1) for each $n$ in $N, V \cap S_{n}$ is open in $S_{n}$, and (2) if $p^{*} \in V$ then $V$ contains $S_{n}$ for all but finitely many $n$. It is not difficult to show that $Y$ is $R$-closed, that $p^{*}$ is the only bad point of $Y$, and that $Y$ has a countable neighborhood base at $p^{*}$.

Assume that for some $M R$ space $X$ there is a finite-to-one mapping $f$ from $X$ onto $Y$.

We first claim that for each $n$ in $N, f^{-1}\left(S_{n}\right)$ is an $M R$ subspace of $X$. For suppose that $m \in N$ and that $\mathscr{B}$ is a regular filterbase on $f^{-1}\left(S_{m}\right)$ having a unique cluster point $x$. If $x \notin f^{-1}\left(p^{*}\right)$ then, since $f^{-1}\left(p^{*}\right)$ is finite, there is some $B^{\prime}$ in $\mathscr{B}$ such that $B^{\prime} \cap f^{-1}\left(p^{*}\right)=\varnothing$; since $f^{-1}\left(S_{m}\right)-f^{-1}\left(p^{*}\right)$ is open in $X$ and closed in $X-f^{-1}\left(p^{*}\right)$, it follows that $\left\{B \in \mathscr{B} \mid B \subseteq B^{\prime}\right\}$ is a regular filterbase in $X$ which must converge to $x$ both in $X$ and in $f^{-1}\left(S_{m}\right)$. If $x \in f^{-1}\left(p^{*}\right)$ then there is some $B^{\prime \prime}$ in $\mathscr{B}$ such that $B^{\prime \prime} \cap\left(f^{-1}\left(p^{*}\right)-\{x\}\right)=\varnothing$; therefore,

$\left\{B \cup U \mid B \in \mathscr{B}, B \subseteq B^{\prime \prime}, U\right.$ is an open neighborhood of $x$ in $\left.X\right\}$

is a regular filterbase on $X$ which must converge to $x$ both in $X$ and in $f^{-1}\left(S_{m}\right)$. Hence, each $f^{-1}\left(S_{n}\right)$ is an $M R$ subspace of $X$.

Now for each $n$ in $N$, let $\mathscr{B}_{n}$ be a nonconvergent regular filterbase on $S_{n}$ having $p^{*}$ as its only cluster point, and let $\mathscr{B}_{n}^{-1}$ denote the collection $\left\{f^{-1}(G) \mid G \in \mathscr{B}_{n}\right\}$. Then for each $n, \mathscr{B}_{n}^{-1}$ is a regular filterbase on $f^{-1}\left(S_{n}\right)$ with each of its cluster points in $f^{-1}\left(p^{*}\right)$ and such that for some neighborhood $W_{n}$ (in $f^{-1}\left(S_{n}\right)$ ) of $f^{-1}\left(p^{*}\right)$ no member of $\mathscr{B}_{n}^{-1}$ is contained in $W_{n}$. Let $q_{1}, \cdots, q_{k}$ denote the points of $f^{-1}\left(p^{*}\right)$ and choose open neighborhoods $V_{1}, \cdots, V_{k}$ of $q_{1}, \cdots, q_{k}$, respectively, such that the closures of the $V_{i}$ 's are disjoint.

We assert that for each $n, f^{-1}\left(S_{n}\right)-\bigcup_{i=1}^{k} \bar{V}_{i} \neq \varnothing$. For assume the contrary, i.e., that for some $m$ in $N, f^{-1}\left(S_{m}\right) \subseteq \bigcup_{i=1}^{k} \bar{V}_{i}$. Since the $\bar{V}_{i}$ 's are disjoint, it follows that each set $\bar{V}_{i} \cap f^{-1}\left(S_{m}\right)$ is both open and closed in $f^{-1}\left(S_{m}\right)$. For each $i(1 \leqq i \leqq k)$ there are two 
possibilities. If $i$ is such that each member of $\mathscr{B}_{m}^{-1}$ intersects $\bar{V}_{i}$ then $\left\{B \cap \bar{V}_{i} \mid B \in \mathscr{B}_{m}^{-1}\right\}$ is a regular filterbase on $f^{-1}\left(S_{m}\right)$ and must converge to $q_{i}$. In this case choose $B_{i}$ to be a member of $\mathscr{B}_{m}^{-1}$ such that $B_{i} \cap \bar{V}_{i} \cong W_{m}$. If $i$ is such that some member of $\mathscr{B}_{m}^{-1}$ misses $\bar{V}_{i}$, choose $B_{i}$ to be such a member of $\mathscr{B}_{m}^{-1}$. Then for each $i$, $B_{i} \cap \bar{V}_{i} \subseteq W_{m}$. Now let $\widetilde{B}$ be a member of $\mathscr{B}_{m}^{-1}$ which lies in $\bigcap_{i=1}^{k} B_{i}$. We have $\bigcup_{i=1}^{k}\left(\widetilde{B} \cap \bar{V}_{i}\right) \cong W_{m}$; i.e., $\widetilde{B} \cong W_{m}$. But since no member of $\mathscr{B}_{m}^{-1}$ is contained in $W_{m}$ this gives us a contradiction.

So for each $n$ we can choose $z_{n}$ to be a point of $f^{-1}\left(S_{n}\right)-\bigcup_{i=1}^{k} \bar{V}_{i}$. Now, for each $n$, let $\mathscr{R}_{n}$ denote the collection of all open neighborhoods in $X$ of the set $\left\{z_{n}, z_{n+1}, z_{n+2}, \cdots\right\}$. Let $\mathscr{R}=\bigcup_{n=1}^{\infty} \mathscr{R}_{n}$. It is not difficult to verify that $\mathscr{R}$ is a regular filterbase on $X$ and that $\mathscr{R}$ can have no cluster point in $X-f^{-1}\left(p^{*}\right)$. But the open set $X-\bigcup_{i=1}^{k} \bar{V}_{i}$ is a member of $\mathscr{R}$ and misses the neighborhood $\bigcup_{i=1}^{k} V_{i}$ of $f^{-1}\left(p^{*}\right)$. Hence, we are forced to the contradictory conclusion that $\mathscr{R}$ has no cluster point.

5. Continuous $M R$ images. In this section we shall make use of the following definition.

Definition 5.1. A regular $T_{1}$ space $X$ is said to be $R$-functionally compact if for each regular $T_{1}$ space $Y$ and each mapping $f$ from $X$ onto $Y, f$ is closed.

The theorem below characterizes $R$-functionally compact spaces and shows their relationship to spaces whose regular $T_{1}$ continuous images are always $M R$. Since (a) $\Rightarrow$ (c) (below), every $R$-functionally compact space is $M R$. However, M. P. Berriozabal and C. F. Blakemore [3] have recently described an example of a nonclosed mapping of an $M R$ space onto a regular $T_{1}$ space, so not every $M R$ space is $R$-functionally compact.

THEOREM 5.2. Let $X$ be a regular $T_{1}$ space. Then conditions (a) and (b), below, are equivalent and either of these implies condition (c).

(a) The space $X$ is $R$-functionally compact.

(b) For each closed set $F$ in $X$ and each regular filterbase $\mathscr{Q}$ for which $F=\cap \mathscr{U}(=\cap \overline{\mathscr{U}}), \mathscr{U}$ is a neighborhood filterbase for $F$.

(c) Every regular $T_{1}$ continuous image of $X$ is $M R$.

Proof. The results (a) $\Rightarrow$ (c) and (b) $\Rightarrow$ (a) can be obtained by arguments essentially the same as those given in the proofs of [4, Theorem 3] and [14, Theorem 2.1], respectively. The proof of $(a) \Rightarrow(b)$, which follows, is a modification of the second half of the 
proof of [4, Theorem 3]. Suppose that $\mathscr{C}$ is a regular filterbase on $X$ and that $F=\cap \mathscr{U}$. Let $W$ be an open neighborhood of $F$. We shall show that some member of $\mathscr{C}$ is contained in $W$. Define $Y$ to be the decomposition of $X$ whose only nondegenerate element is $F$, and let $f$ be the natural projection of $X$ onto $Y$. We choose a base $\mathscr{Y}$ for a topology on $Y$ as follows: a subset $V$ of $Y$ is in $\mathscr{V}$ if and only if (1) $f^{-1}(V)$ is an open subset of $X-F$, or (2) $f^{-1}(V) \in \mathscr{K}$. It is easily seen that $Y$ is Hausdorff. Since the restriction of $f$ to $X-F$ is a homeomorphism onto $Y-f(F)$, it is clear that $Y$ is regular at each point of $Y-f(F)$. For each $V$ in $\mathscr{V}$ containing $f(F), f^{-1}\left(\mathrm{Cl}_{Y} V\right)=\mathrm{Cl}_{Y} f^{-1}(V)$, and since $\mathscr{U}$ is regular this implies that $Y$ is regular at $f(F)$. Thus, $Y$ is a regular $T_{1}$ space. It now follows from the $R$-functional compactness of $X$ that $f(X-W)$ is closed in $Y$ and, therefore, that $Y-f(X-W)$ is an open neighborhood of $f(F)$. So for some $V$ in $\mathscr{V}$ we have $f\left(F^{\prime}\right) \in V$ and $V \subseteq Y-f(X-W)$. Consequently, $f^{-1}(V) \in \mathscr{U}$ and $f^{-1}(V) \subseteq W$.

\section{QUESTIONS 5.3.}

(1) Does condition (c) of Theorem 5.2 imply condition (a)?

(2) Is every $R$-functionally compact space necessarily compact?

An affirmative answer to 5.3(2) would imply that every regular $T_{1}$ space having each of its closed sets $R$-closed is compact, thus answering a question of B. Banaschewski (see [2, Problem 14]). However, we conjecture that the answer to 5.3(2) is no.

Addendum. It has come to our attention that at about the same time that our research for this paper was being completed, R. F. Dickman, Jr., and T. O. Vinson, Jr., obtained results very similar to our Corollaries 2.10 and 3.9. Specifically, they proved (1) that every $H$-closed space is the image, under an open mapping, of an $M H$ space, and (2) that the inverse limit of a system of $H$-closed spaces is $H$-closed if all bonding maps are open and onto.

\section{REFERENCES}

1. A. Arhangel'skij, Some types of factor mappings and the relations between classes topological spaces, Dokl. Akad. Nauk. SSSR, 153 (1963), 743-746. (=Soviet Math. Dokl., 4 (1963), 1726-1729).

2. M. P. Berri, J. R. Porter, R. M. Stephenson, Jr., A survey of minimal topological spaces, Proc. Kanpur Top. Conf. 1968, General Topology and its Relations to Modern Analysis and Algebra III (Academic Press, NY., 1970)

3. M. P. Berriozabal (Berri) and C. F. Blakemore, The regular continuous image of a minimal regular space is not necessarily minimal regular, Proc. Amer. Math. Soc., 51 (1975), 453-454.

4. R. F. Dickman, Jr. and A. Zame, Functionally compact spaces, Pacific J. Math., 
31 (1969), 303-311.

5. James Dugundji, Topology, Allyn and Bacon, Boston (1966).

6. Douglas Harris, Katétov extension as a functor, Math. Ann., 193 (1971), 171-175.

7. Stephen Hechler, On a notion of weak compactness in non-regular spaces, Studies in General Topology (1975), Academic Press, 215-237.

8. C. T. Liu and G. E. Strecker, Concerning almost real compactifications, Czech. Math. J., 22 (1972), 181-190.

9. Dix H. Pettey, A minimal regular space that is not strongly minimal regular, Canad. J. Math., 28 (1976), 875-878.

10. C. T. Scarborough and A. H. Stone, Products of nearly compact spaces, Trans. Amer. Math. Soc., 124 (1966), 131-147.

11. R. M. Stephenson, Jr., Not every minimal Hausdorff space is e-compact, Proc. Amer. Math. Soc., 52 (1975), 381-389.

12. - Product spaces and the Stone-Weierstrass theorem, General Topology and Appl., 3 (1973), 77-79.

13. - Two R-closed spaces, Canad. J. Math., 24 (1972), 286-292.

14. S. W. Willard, Functionally compact spaces, c-compact spaces and mappings of minimal Hausdorff spaces, Pacific J. Math., 38 (1971), 267-272.

Received May 22, 1975 and in revised form March 24, 1976.

UNIVERSITY OF MissourI

Columbia, MO 65201 


\section{PACIFIC JOURNAL OF MATHEMATICS}

\section{EDITORS}

RICHARD ARENS (Managing Editor)

University of California

Los Angeles, CA 90024

Charles W. Curtis

University of Oregon

Eugene, OR 97403

C. C. MOORE

University of California

Berkeley, CA 94720

\section{J. DugundJI}

Department of Mathematics

University of Southern California

Los Angeles, CA 90007

R. FinN and J. Milgram

Stanford University

Stanford, CA 94305

ASSOCIATE EDITORS
E. F. BECKENBACH
B. H. NeumanN
F. WOLF
K. YOSHIDA

\section{SUPPORTING INSTITUTIONS}

UNIVERSITY OF BRITISH COLUMBIA CALIFORNIA INSTITUTE OF TECHNOLOGY

UNIVERSITY OF CALIFORNIA

MONTANA STATE UNIVERSITY

UNIVERSITY OF NEVADA, RENO

NEW MEXICO STATE UNIVERSITY

OREGON STATE UNIVERSITY

UNIVERSITY OF OREGON

OSAKA UNIVERSITY

\author{
UNIVERSITY OF SOUTHERN CALIFORNIA \\ STANFORD UNIVERSITY \\ UNIVERSITY OF HAWAII \\ UNIVERSITY OF TOKYO \\ UNIVERSITY OF UTAH \\ WASHINGTON STATE UNIVERSITY \\ UNIVERSITY OF WASHINGTON \\ AMERICAN MATHEMATICAL SOCIETY
}

The Supporting Institutions listed above contribute to the cost of publication of this Journal, but they are not owners or publishers and have no responsibility for its content or policies.

Mathematical papers intended for publication in the Pacific Jaurnal of Mathematics should be in typed form or offset-reproduced, (not dittoed), double spaced with large margins. Please do not use built up fractions in the text of your manuscript. You may however, use them in the displayed equations. Underline Greek letters in red, German in green, and script in blue. The first paragraph or two must be capable of being used separately as a synopsis of the entire paper. Items of the bibliography should not be cited there unless absolutely necessary, in which case they must be identified by author and Journal, rather than by item number. Manuscripts, in triplicate, may be sent to any one of the editors. Please classify according to the scheme of Math. Reviews, Index to Vol. 39. All other communications should be addressed to the managing editor, or Elaine Barth, University of California, Los Angeles, California, 90024.

The Pacific Journal of Mathematics expects the author's institution to pay page charges, and reserves the right to delay publication for nonpayment of charges in case of financial emergency.

100 reprints are provided free for each article, only if page charges have been substantially paid. Additional copies may be obtained at cost in multiples of 50 .

The Pacific Journal of Mathematics is issued monthly as of January 1966. Regular subscription rate: $\$ 7200$ a year (6 Vols., 12 issues). Special rate: $\$ 36.00$ a year to individual members of supporting institutions.

Subscriptions, orders for back numbers, and changes of address should be sent to Pacific Journal of Mathematics, 103 Highland Boulevard, Berkeley, California, 94708.

PUBLISHED BY PACIFIC JOURNAL OF MATHEMATICS, A NON-PROFIT CORPORATION

Printed at Kokusai Bunken Insatsusha (International Academic Printing Co., Ltd.). 8-8, 3-chome, Takadanobaba, Shinjuku-ku, Tokyo 160, Japan.

Copyright (C) 1975 by Pacific Journal of Mathematics Manufactured and first issued in Japan 


\section{Pacific Journal of Mathematics}

\section{Vol. 71, No. $2 \quad$ December, 1977}

Krishnaswami Alladi and Paul Erdős, On an additive arithmetic

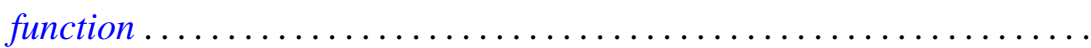

James Bailey and Dale Rolfsen, An unexpected surgery construction of a

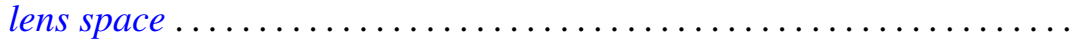

Lawrence James Brenton, On the Riemann-Roch equation for singular

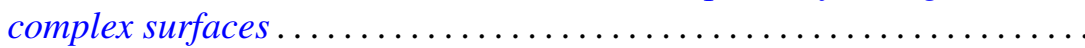

James Glenn Brookshear, Projective ideals in rings of continuous

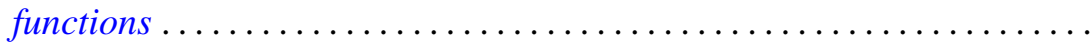

Lawrence Gerald Brown, Stable isomorphism of hereditary subalgebras of

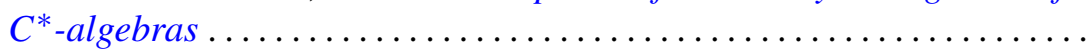

Lawrence Gerald Brown, Philip Palmer Green and Marc Aristide Rieffel, Stable isomorphism and strong Morita equivalence of $C^{*}$-algebras....

N. Burgoyne, Robert L. Griess, Jr. and Richard Lyons, Maximal subgroups

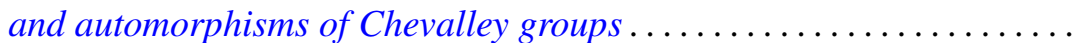
365

Yuen-Kwok Chan, Constructive foundations of potential theory .... 405

Peter Fletcher and William Lindgren, On $w \Delta$-spaces, $w \sigma$-spaces and

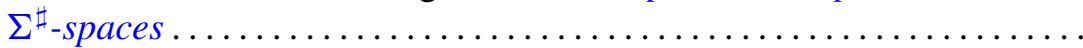

Louis M. Friedler and Dix Hayes Pettey, Inverse limits and mappings of

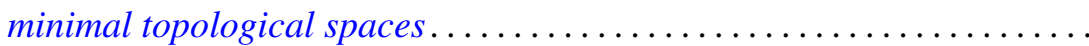

Robert E. Hartwig and Jiang Luh, A note on the group structure of unit regular ring elements.

I. Martin (Irving) Isaacs, Real representations of groups with a single involution ...

Nicolas P. Jewell, The existence of discontinuous module derivations . .

Antonio M. Lopez, The maximal right quotient semigroup of a strong semilattice of semigroups .......................

Dennis McGavran, $T^{n}$-actions on simply connected $(n+2)$-manifolds

Charles Anthony Micchelli and Allan Pinkus, Total positivity and the exact $n$-width of certain sets in $L^{1}$.

Barada K. Ray and Billy E. Rhoades, Fixed point-theorems for mappings with a contractive iterate .......................

Fred Richman and Elbert A. Walker, Ext in pre-Abelian categories. .

Raymond Craig Roan, Weak* generators of $H^{\infty}$ and $l^{1}$..

Saburou Saitoh, The exact Bergman kernel and the kernels of Szegö type...

Kung-Wei Yang, Operators invertible modulo the weakly compact 